\title{
Bacteriemia por Campylobacter jejuni en un paciente con infección por VIH en etapa SIDA
}

\author{
Felipe Bravo', Alejandra Céspedes², Pedro Morales² y Leonardo Chanqueo²
}

\section{Campylobacter jejuni bacteremia in a patient with HIV infection in AIDS stage}

Bacteremia is an atypical presentation of Campylobacter jejuni infection and it is more frequent in patients with advanced inmunodepression due to HIV or other sistemic diseases. Because of the highly active antiretroviral therapy, in the last decades the number of cases had declined. We report a case of a homeless woman with HIV in $\mathrm{C} 3$ stage who was diagnosed with the bacteremia during her hospitalization for pulmonary tuberculosis, and a brief review of $C$. jejuni bacteremia in HIV patients.

Keywords: Bacteremia; Campylobacter jejuni; HIV.

Palabras clave: Bacteriemia; Campylobacter jejuni; VIH.

\section{Introducción}

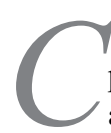

ampylobacter jejuni es uno de los principales patógenos bacterianos causantes de diarrea aguda en la población general. La infección es adquirida principalmente por el consumo de alimentos o agua contaminados, y con menos frecuencia del contacto directo con animales ${ }^{1}$. El cuadro clínico clásico es el de una gastroenteritis aguda, que suele resolverse espontáneamente en personas inmunocompetentes. Puede cursar con otras manifestaciones digestivas como colecistitis, o extradigestivas como artritis o endocarditis; y complicaciones posteriores como síndrome de intestino irritable y síndrome de Guillain Barré ${ }^{2}$. De manera muy infrecuente se puede presentar con una bacteriemia, cuyo curso clínico puede ser más grave en pacientes con co-morbilidades como infección por VIH, daño hepático crónico, patologías hematológicas en quimioterapia, o infección por el virus de hepatitis $\mathrm{C}^{2}$.

En nuestro país, se han reportado casos aislados de bacteriemias por Campylobacter spp., principalmente $C$. fetus, en pacientes con co-morbilidades hematológicas, cirrosis hepática y enfermedades cardiovasculares, pero no se han comunicado casos en pacientes infectados por $\mathrm{VIH}^{3}$.

Se presenta un caso de bacteriemia por C. jejuni en una paciente con infección por VIH en etapa SIDA y se realiza una revisión del tema en la literatura médica.

\section{Caso clínico}

Mujer de 27 años, con discapacidad intelectual y trastorno del aprendizaje, que vivía en situación de calle en la comuna de Santiago. Tenía antecedente de consumo de pasta base, cocaína y marihuana. Se le había diagnosticado una tuberculosis (TBC) pulmonar e infección por VIH, con exámenes en ese momento: CD4 87 céls $/ \mathrm{mm}^{3}$, carga viral (CV) 17.381 copias de ARN viral/ml (Log 4,24 copias $/ \mathrm{ml}$ ). La paciente no asistió a controles posteriores por lo que no completó el tratamiento antituberculoso, y tampoco inició terapia anti-retroviral (TARV).

Seis meses después del diagnóstico es llevada por su madre al Servicio de Urgencia de nuestro hospital por disnea progresiva, tos con expectoración mucopurulenta, sensación febril y diarrea acuosa, sin elementos patológicos. Al ingreso se encontraba consciente, deshidratada, enflaquecida, con signos vitales: FC 87 lpm, PA 93/50, $\mathrm{T}^{\circ} 36 \mathrm{C}$, saturación $97 \%$ con $\mathrm{FiO}_{2}$ ambiental. Al examen físico segmentario destacó un murmullo pulmonar disminuido con crépitos bilaterales, con un abdomen blando, no doloroso. La radiografía de tórax evidenció imágenes cicatriciales que comprometían el lóbulo superior derecho, de relleno alveolar en lóbulo medio derecho y, en el campo pulmonar izquierdo, imágenes bulosas de gran tamaño. Se realizaron baciloscopías de expectoración que resultaron positivas $(+++)$.

La paciente se hospitalizó con diagnóstico de una TBC pulmonar, insuficiencia respiratoria aguda y VIH/SIDA etapa C3. Se indicó reinicio de esquema primario de tratamiento anti-tuberculoso, ajustado por peso $<45 \mathrm{~kg}, \mathrm{y}$ profilaxis con cotrimoxazol $400 \mathrm{mg}$ (trimetoprim $160 \mathrm{mg}$ / sulfametoxazol $800 \mathrm{mg}$ ). En los exámenes destacó una anemia ( $\mathrm{Hb} 8,5 \mathrm{mg} / \mathrm{dl})$ normocítica normocrómica, leucocitos 5.800 céls $/ \mathrm{mm}^{3}$, plaquetas 323.000 , VHS $90 \mathrm{~mm} / \mathrm{h}$, función renal normal, proteína C reactiva (PCR) $61,5 \mathrm{mg} / 1$ (VN: <5), albúmina 2,5 mg/dl, pruebas hepáticas normales a excepción de fosfatasa alcalina en $296 \mathrm{U} / \mathrm{L}$ (VN: 40-150), recuento de CD4 113 céls $/ \mathrm{mm}^{3}$ y CV 198.977 copias/ml. Evolucionó los dos primeros días con fiebre de $39^{\circ} \mathrm{C}$, sin deterioro clínico, sin disnea, ni diarrea.
'Departamento de Medicina Interna Occidente. Facultad de Medicina, Universidad de Chile. ${ }^{2}$ Hospital San Juan de Dios, Santiago, Chile.

Los autores declaran no tener conflictos de interés.

Recibido: 15 de octubre de 2018 Aceptado: 8 de agosto de 2019

Correspondencia a: Felipe Bravo B. felipeb84@gmail.com. 
Al tercer día de hospitalización por persistencia de fiebre y taquicardia se decidió realizar hemocultivos e iniciar ceftriaxona $2 \mathrm{~g}$ iv. Se realizó una TC de tórax y cerebro, látex de Criptococcus en sangre y hemocultivo para micobacterias. En los exámenes destacó un alza de PCR a $202 \mathrm{mg} / \mathrm{L}$ y leucocitos a 10.550 céls $/ \mathrm{mm}^{3}$. Posteriormente, se informó un hemocultivo positivo a las $52 \mathrm{~h}$ para un bacilo gramnegativo (probable bacilo curvo) por lo cual se decidió mantener terapia antimicrobiana con ceftriaxona.

El hemocultivo se subcultivó según protocolo local en agar sangre Columbia, chocolate y MacConkey. El cultivo presentó un crecimiento pobre en agar sangre Columbia y agar chocolate a las $72 \mathrm{~h}$ de incubación a $37^{\circ} \mathrm{C}$ con $5 \%$ $\mathrm{CO} 2$; no creció en agar MacConkey. Por las características de la colonia, muy inhibida, no se logró realizar pruebas básicas. Se realizó análisis de la colonia (del agar sangre Columbia) por MALDI-TOF MS (VITEK MS ${ }^{\circledR}$ ) cuyo resultado concluyó Campylobacter jejuni, en dos ocasiones con porcentaje de certeza de $99,9 \%$, sin identificación de subespecie. La cepa fue notificada y enviada al Instituto de Salud Pública (ISP) para confirmación y estudio de susceptibilidad.

Al quinto día de tratamiento con ceftriaxona, por persistencia de fiebre diaria hasta $38,5^{\circ} \mathrm{C}$, aunque sin deterioro clínico, y ante el informe de laboratorio de C. jejuni se cambió la terapia a ciprofloxacina $400 \mathrm{mg}$ cada $12 \mathrm{~h}$ iv. asociado a azitromicina $500 \mathrm{mg}$ al día vía oral (no hubo disponibilidad de azitromicina iv.). La paciente evolucionó con disminución de la curva febril y de parámetros inflamatorios, y no tuvo diarrea durante la hospitalización. Se realizó control de hemocultivos a los cinco días de tratamiento, que fueron negativos, completando 14 días de la terapia antibacteriana descrita. El hemocultivo para micobacterias fue negativo a los 42 días de incubación. Al término del tratamiento antimicrobiano, se obtuvo el resultado definitivo del ISP que confirmó, por epsilometría, C. jejuni resistente a ciprofloxacina, y sensible a macrólidos y tetraciclina. Al mes del inicio de la terapia antituberculosa, se inició la TARV, evolucionando en buenas condiciones.

\section{Discusión}

La bacteriemia asociada a Campylobacter spp. es muy poco frecuente ${ }^{4} \mathrm{y}$ ha ido en disminución los últimos años. La frecuencia y especie predominante depende según la localización geográfica y nivel de desarrollo económico, donde la infección gastrointestinal por Campylobacter spp es más alta ${ }^{5}$ Aunque en algunas series de casos predomina $C$. fetus ${ }^{6}$ hasta $50 \%$ en bacteriemia, en otras C. jejuni y C. coli $^{4,7}$ son los principales causales, hasta en 1,5 por cada 1000 casos de diarrea, con $28 \%$ de los casos con co-morbilidades subyacentes. La bacteriemia por $C$. jejuni es más frecuente en aquellos pacientes con co-morbilidades e inmunodepresión, con una mortalidad de $10 \%$ atribuible a la bacteriemia ${ }^{8}$.

Tee y cols. en un análisis retrospectivo de 10 años en un hospital australiano ${ }^{9}$ reportaron una incidencia de $8,3 \%$ de bacteriemias en pacientes con infección por VIH (media de CD4 184 céls $/ \mathrm{mm}^{3}$ ) con cuadro febril y diarrea aguda por $C$. jejuni, mientras que $1,6 \%$ en pacientes sin VIH. En estos últimos, un factor de riesgo importante fue viajar al sudeste asiático. Llamativamente, un $89 \%$ de los pacientes con infección por VIH cursó con manifestaciones extraintestinales descritas como consolidaciones transitorias en parches en radiografías de tórax; uno de los pacientes tuvo celulitis. Fallecieron tres pacientes, uno de los cuales tuvo una neumonía y enfermedad diseminada por C. jejuni (con aislamiento post-mortem en tejido de linfático y pulmón). Los otros dos fallecidos cursaron con una diarrea crónica y enteritis persistente por C. jejuni, recibiendo múltiples esquemas antimicrobianos.

Manfredi y cols. describieron una disminución de los casos de enfermedad grave causada por Campylobacter spp. ${ }^{10}$ en una revisión de 16 pacientes con VIH (13 fueron C. jejuni). Todas las bacteriemias habían ocurrido antes de 1997, y estaban claramente asociadas con recuentos de CD4 bajos y etapa SIDA. Se evidenció una alta resistencia a cefalosporinas de primera generación $(93,7 \%)$, pero $100 \%$ de sensibilidad a quinolonas, $62 \%$ a macrólidos y $75 \%$ a gentamicina. Los tratamientos antimicrobianos fueron diversos, con una duración entre 8 a 18 días y una alta tasa de recaídas en los pacientes antes de la era de TARV, con un caso letal. Similares hallazgos fueron reportados en un hospital terciario de Madrid (11), donde la inmunodepresión por VIH estaba presente en $23,4 \%$ de los pacientes con bacteriemias por $C$. jejuni. La mortalidad fue mayor en enfermos con VIH que en aquellos no infectados (33 vs 10\%). La incidencia de casos fue mayor previa a la era de instauración de TARV (27,5 vs 14,3\%). En la actualidad, la bacteriemia por $C$. jejuni en pacientes con infección por VIH es infrecuente, con prevalencia de $1,5 \%$ del total de bacteriemias en pacientes adultos con VIH hospitalizados ${ }^{12}$.

En nuestro país, Fica y cols. ${ }^{3}$ comunicaron dos casos de bacteriemias por $C$. jejuni (y cinco con C. fetus, dos de ellos con resultado fatal) entre 1986 y 2010, y aunque no hubo pacientes con VIH, todos presentaban otras comorbilidades crónicas. La mayoría de las bacteriemias ocurrieron luego del año 2000, a pesar de que el uso de método de hemocultivos automatizados se había iniciado en 1996, por lo cual los autores sugieren que el aumento de éstas no estaría dado por el sistema utilizado y podría deberse a los cambios en la industria alimentaria en Chile. Hubo además, un aumento en la detección de Campylobacter spp. en coprocultivos entre 2004 y 2010 desde menos de $0,5 \%$ a más de $2 \%$. Esta cifra es concordante con los hallazgos descritos por Chanqueo y cols. en estudio 
de patógenos entéricos entre 2002 y 2004 en la Red de Salud UC en Santiago ${ }^{13}$.

En nuestro caso clínico, se conjugan dos factores de riesgo para desarrollar la bacteriemia por $C$. jejuni: la inmunodepresión intensa asociada al VIH sin el uso de TARV y, aunque no se pudo certificar exactamente la ingesta alimentaria de la paciente, es probable el consumo de alimentos de riesgo debido a que se encontraba viviendo en situación de calle.

Tradicionalmente se describe que la inmunidad humoral es crucial en la protección contra la enfermedad por Campylobacter spp. ${ }^{14}$, lo que explica la alta frecuencia en pacientes con déficit de inmunoglobulinas. Sin embargo, las alteraciones globales en el sistema inmune en pacientes con VIH, con predominio del deterioro de la inmunidad celular mediada por células $\mathrm{T}$, sumado a la falla en la inmunidad de mucosas e integridad de barrera intestinal, incrementan el riesgo de infecciones por bacterias entéricas, C. jejuni entre ellos. Campylobacter jejuni posee factores de virulencia, como motilidad flagelar y una cápsula polisacárida que favorece la evasión del sistema inmune, la adherencia y la invasión de células epiteliales ${ }^{15}$. Por ende, la recuperación inmunológica y de niveles de CD4 secundaria a una TARV eficaz es de vital importancia para el control de la infección por este agente. En el caso de nuestra paciente, luego del inicio de TARV no se objetivó una recaída de la bacteriemia durante su hospitalización, ni otras complicaciones extradigestivas. Asimismo, en el seguimiento ambulatorio se evidenció la recuperación de CD4 mayor a 200 céls $/ \mathrm{mm}^{3}$ y el descenso de la CV a niveles indetectables, y no se evidenciaron recurrencias de la bacteriemia. Esto puede reafirmar la importancia de la mejoría de la inmunodepresión en la disminución de la frecuencia de infecciones graves por C. jejuni.

En cuanto al tratamiento, generalmente en personas inmunocompetentes las infecciones por $C$. jejuni no requieren terapia antimicrobiana, ya que son autolimitadas ${ }^{16}$ y requieren solo manejo de soporte (hidratación, dieta, analgesia). En pacientes inmunocomprometidos; sin embargo, la bacteriemia requiere una terapia antimicrobiana eficaz. Anteriormente las quinolonas ${ }^{17}$ habían sido una de las terapias de elección, situación que ha cambiado debido al incremento en la última década de resistencia a esta familia de antibacterianos ${ }^{18}$, con tasas hasta $42 \%$ en Latinoamérica y Europa, e inclusive hasta más de $80 \%$ de resistencia en países asiáticos.

La resistencia a macrólidos es más baja, entre 1,1 a $4 \%{ }^{19,20}$; los aminoglicósidos (gentamicina en particular) también mantienen buena actividad sobre $C$. jejuni, y pueden ser parte de la terapia ${ }^{3,18}$. Debido a la presencia de $\beta$-lactamasas encontradas en $C$. jejuni, el uso de penicilinas no es recomendado. Por otra parte, el tratamiento con cefalosporinas de tercera generación se ha asociado a mayor mortalidad en casos de bacteriemia por Campylo- bacter spp., exceptuando C. fetus ${ }^{6}$. Los carbapenémicos son una excelente alternativa empírica especialmente en cuadros de mayor gravedad con bacteriemia ${ }^{6}$, hasta obtener el resultado de susceptibilidad definitiva.

La recomendación de terapia de los $C D C$, NIH y HIV Medicine Association of the Infectious Diseases Society of America en bacteriemia por Campylobacter spp. es guiar la terapia antimicrobiana según el estudio de susceptibilidad, y en caso de sensibilidad a quinolonas, el tratamiento sugerido es ciprofloxacina (oral o iv) por 14 días. Adicionar un aminoglicósido podría ser prudente para limitar la emergencia de resistencia. Por último, no recomiendan el uso de azitromicina (Tabla 1$)^{21}$.

Respecto a la susceptibilidad antimicrobiana de $C$. jejuni en Chile, García y cols. ${ }^{22}$ reportaron en 73 muestras de deposiciones en la Red Salud UC en Santiago, 32,4\% resistencia a ciprofloxacina, 6,4\% a ampicilina, y no detectaron resistencia a macrólidos.

En el caso de nuestra paciente se definió cobertura antimicrobiana con ciprofloxacina y azitromicina, considerando la estabilidad clínica de la paciente, a pesar de los episodios de fiebre diarios, pero sin cursar con sepsis y/o shock séptico. Para objetivar la resolución de la bacteriemia y evaluar la respuesta a la terapia, considerando la falta de estudio de susceptibilidad disponible a nivel local para el agente, se realizaron hemocultivos de control, lo cual se encuentra sugerido en el seguimiento en infección de sitios estériles por Campylobacter spp. ${ }^{23}$.

Por último, cabe destacar el rol de de MALDI-TOF en la identificación de $C$. jejuni en este caso. Usualmente la identificación y diferenciación de Campylobacter spp. es un desafío debido a su pobre actividad bioquímica y compleja taxonomía, además el cultivo específico es de alto costo, requiere medios selectivos (en el caso de nuestro centro, no disponible) e incubación en microaerofilia a $42^{\circ 13}$. En este sentido, se ha reportado que MALDI-TOF presenta alto rendimiento en la identificación de especies de Campylobacter spp. con discordancia de $0,4 \%$ respecto al estándar de oro ${ }^{23}$, sumado además al beneficio de la rapidez de detección.

En conclusión, a pesar de que la infección gastrointestinal por Campylobacter spp. es común, la bacteriemia por C. jejuni es un cuadro de baja frecuencia, con variaciones dependiendo del área geográfica. Las observaciones descritas a través de los últimos años describen una disminución en la ocurrencia de bacteriemias por C. jejuni en pacientes con VIH, probablemente debido al mayor acceso a la TARV, aunque persisten con mayor frecuencia que la población general sin VIH. La susceptibilidad antimicrobiana, especialmente en relación a quinolonas, tal como lo evidenciado en nuestro caso con resistencia a ciprofloxacina, es un factor importante a considerar en la elección de la terapia empírica, más aun considerando la posibilidad de recaídas y la mayor mortalidad en relación a pacientes sin infección por VIH. 


\begin{tabular}{l}
$\begin{array}{l}\text { Tabla 1. Recomendaciones de manejo de bacteriemia por } \boldsymbol{C} \text {. } \\
\text { jejuni en pacientes con VIH (adaptado de referencias } 6 \text { y 21) }\end{array}$ \\
\hline - Guiar antibioterapia por estudio de susceptibilidad \\
- Terapia empírico en caso de gravedad: \\
*ciprofloxacina + un aminoglicósido (gentamicina) \\
*carbapenémicos (imipenem, meropenem) \\
- No utilizar cefalosporinas de tercera generación \\
- Duración de terapia 14 días. Extender a más de dos, hasta seis \\
semanas si hay recurrencia de bacteriemia \\
- No retrasar inicio de TARV (IRIS no ha sido descrito) \\
\hline $\begin{array}{l}\text { Abreviaturas: TARV: terapia anti-retroviral IRIS: síndrome inflamato- } \\
\text { rio de reconstitución inmune (por siglas en inglés). }\end{array}$
\end{tabular}

\section{Resumen}

La bacteriemia es una presentación atípica de la infección por Campylobacter jejuni, y es más frecuente en pacientes con inmunodepresión avanzada debido al VIH u otras enfermedades sistémicas. Debido a la terapia anti-retroviral, en las últimas décadas el número de casos ha disminuido. Presentamos el caso de una mujer en situación de calle, con VIH en etapa C3, que cursó con una bacteriemia por $C$. jejuni durante su hospitalización por una tuberculosis pulmonar. Realizamos una breve revisión de bacteriemia por $C$. jejuni en pacientes con VIH.

\section{Referencias bibliográficas}

1.- Kaakoush N O, Castaño-Rodríguez N, Mitchell H M, Man S M. Global epidemiology of Campylobacter infection. Clin Microbiol Rev 2015; 28: 687-720. doi: 10.1128/CMR.0000615

2.- Mori T, Hasegawa N, Sugita K, Shinjoh M, Nakamoto N, Shimizu T, et al. Clinical features of bacteremia due to Campylobacter jejuni. Intern Med 2014; 53: 1941-4.

3.- Fica A, Porte L, Braun S, Veas N, Pavez C, Dabanch J, et al. Bacteriemias e infección endovascular por Campylobacter spp: nuestra experiencia en un cuarto de siglo de historia. Rev Chilena Infectol 2011; 28: 211-6

4.- Feodoroff A, Lauhio A, Ellstrom P, Rautellin H. A nationwide study of Campylobacter jejuni and Campylobacter coli bacteremia in Finland over a 10-year period, 1998-2007, with special reference to clinical characteristics and antimicrobial susceptibility. Clin Infect Dis 2011; 53: e99-e106. doi: 10.1093/cid/cir509.

5.- Kownhar H, Shankar E, Rajan R, Vengatesan A, Rao UA. Prevalence of Campylobacter jejuni and enteric bacterial pathogens among hospitalized HIV infected versus non-HIV infected patients with diarrhoea in southern India. Scand J Infect Dis 2007; 39: 862-6.

6.- Pacanowski J, Lalande V, Lacombe K, Boudraa C, Lesprit P, Legrand P, et al. Campylobacter bacteremia: clinical features and factors associated with fatal outcome. Clin Infect Dis 2008; 47: 790-79. doi: 10.1086/591530.

7.- Skirrow M B, Jones D M, Sutcliffe E, Benjamin J. Campylobacter bacteremia in England and Wales, 1981-91. Epidemiol Infect 1993; 110: 567-73.

8.- Font C, Cruceta A, Moreno A, Miró O, Coll-Vinent B, Almela M, et al. A study of 30 patients with bacteremia due to Campylobacter spp. Med Clin (Barc) 1997; 9: 336-40.

9.- Tee W, Mijch A. Campylobacter jejuni bacteremia in human immunodeficiency virus (HIV) infected and non-HIV-infected patients:
Comparison of clinical features and review. Clin Infect Dis 1998; 26: 91-6.

10.- Manfredi R, Calza L, Chiodo F. Enteric and disseminated Campylobacter species infection during HIV disease: a persisting but significantly modified association in the HAART Era. Am J Gastroenterol 2002; 97 : 510-1.

11.- Fernández-Cruz A, Muñoz P, Mohedano R, Valerio M, Marín M, Alcalá L, et al. Campylobacter bacteremia: clinical characteristics, incidence, and outcome over 23 years. Medicine (Baltimore) 2010; 89: 319-30. doi: 10.1097/MD.0b013e3181f2638d.

12.- Huson M, Stolp S, van der Poll T, Grobusch M. Community-acquired bacterial bloodstream infections in HIV-infected patients: a systematic review. Clin Infect Dis 2014; 58: 79-92. doi: 10.1093/cid/cit596.

13.- Chanqueo L, García P, León E, Blu A. Evaluación de la tinción de Hucker para la búsqueda rutinaria de Campylobacter sp en el estudio de un síndrome diarreico agudo. Rev Chilena Infectol 2005; 22 (3): 242-6

14.- Janssen R, Krogfelt K, Cawthraw S, Van Pelt $\mathrm{W}$, Wagenaar J. Host-pathogen interactions in Campylobacter infections: the host perspective. Clin Microbiol Rev 2008; 21: 505-18.

15.- Keo T, Collins J, Kunwar P, Blaser, MJ, Iovine NM. Campylobacter capsule and lipooligosaccharide confer resistance to serum and cationic antimicrobials. Virulence 2011; 2: 30-40.

16.- Allos B. Campylobacter jejuni infections: update on emerging issues and trends. Clin Infect Dis 2001; 32: 1201-6.

17.- Kirckpatrick B D, Tribble D R. Update on human Campylobacter jejuni infections. Curr Opin Gastroenterol 2011; 27: 1-7. doi: 10.1097/ MOG.0b013e3283413763.

18.- Streit J M, Jones R N, Toleman M A, Stratchounski L S, Fritsche T R. Prevalence and antimicrobial susceptibility patterns among gastroenteritis-causing pathogens recovered in Europe and Latin America and Salmonella isolates recovered from bloodstream infections in North America and Latin America: report from the SENTRY Antimicrobial Surveillance Program (2003). Int J of Antimicrob Agents 2006; 27: 367-75.

19.- Zhou J, Zhang M, Yang W, Fang Y, Wang G, Hou F. A seventeen-year observation of the antimicrobial susceptibility of clinical Campylobacter jejuni and the molecular mechanisms of erythromycinresistant isolates in Beijing, China. Int J Infec Dis 2016; 42: 28-33. doi: 10.1016/j. ijid.2015.11.005.

20.- Lehtopolku M, Nakari U M, Kotilainen P, Huovinen P, Siitonen A, Hakanen A J. Antimicrobial susceptibilities of multidrugresistant Campylobacter jejuni and C. coli strains: in vitro activities of 20 antimicrobial agents. Antimicrob Agents Chemother 2010; 54: 1232-6.

21.- Panel on Opportunistic Infections in HIVInfected Adults and Adolescents. Guidelines for the prevention and treatment of opportunistic infections in HIV-infected adults and adolescents: recommendations from the Centers for Disease Control and Prevention, the National Institutes of Health, and the HIV Medicine Association of the Infectious Diseases Society of America. Disponible en http:// aidsinfo.nih.gov/contentfiles/lvguidelines/ adult_oi.pdf; I-1 a I-9. (Acceso: 3 de junio de 2019).

22.- García P, Valenzuela N, Rodríguez V, León E, Fernández H. Susceptibilidad antimicrobiana de Campylobacter jejuni aislado de coprocultivos en Santiago de Chile. Rev Chilena Infectol 2009; 26: 511-4.

23.- Bessede E, Solecki O, Sifré E, Labadi L, Mégraud F. Identification of Campylobacter species and related organisms by matrix assisted laser desorption ionization-time of flight (MALDI-TOF) mass spectrometry. Clin Microbiol Infect 2011; 17: 1735-9. doi: 10.1111/j.1469-0691.2011.03468.x. 